\title{
Co-Amoxiclav as empiric treatment of UTI in children: importance of surveillance in ensuring optimal empiric treatment choice
}

\author{
James Trayer ${ }^{1} \cdot$ Michael Horgan $^{1} \cdot$ Anna-Rose Prior $^{2} \cdot$ Martin Ryan $^{3} \cdot$ Montasser Nadeem $^{1,4}$ (D)
}

Received: 7 March 2021 / Accepted: 12 August 2021 / Published online: 22 August 2021

(c) The Author(s) 2021

\begin{abstract}
Background Urinary tract infections are common and require prompt treatment. Objective To examine the resistance rates of co-amoxiclav in children with urinary tract infection and whether antimicrobial resistance is influenced by other variables. Methods The records and antibiotic susceptibility data of 209 patients admitted with symptomatic urinary tract infection between January 2018 and December 2019 were reviewed. Results We examined 209 patients [mean (SD) age 23.73 (32.86) months], of whom 176 (84.2\%) had first urinary tract infection. Escherichia coli was isolated in 190 (90.1\%). Uropathogens were sensitive to co-amoxiclav in $47.8 \%$ of patients and gentamicin in $95.2 \%$. Combined co-amoxiclav with gentamicin demonstrated antimicrobial sensitivity in $96.2 \%$. Antimicrobial resistance was associated with longer hospital stay $(p$-value $<0.02)$. An association was identified between co-amoxiclav resistance and recurrent urinary tract infections. Uropathogens were resistant to co-amoxiclav in 80/176 (45.5\%) and 29/33 (87.9\%) patients with first and recurrent urinary tract infections, respectively ( $p$-value 0.001 ). No link was observed between antimicrobial resistance and atypical urinary tract infection. Conclusion Approximately half of children in this cohort had urinary tract infection due to uropathogens resistant to co-amoxiclav. Co-amoxiclav resistance is associate with recurrent infections and longer hospital stays. A combination of co-amoxiclav and gentamicin demonstrates $>96 \%$ susceptibility.
\end{abstract}

Keywords Antibiotic resistance $\cdot$ General Paediatric $\cdot$ Nephrology $\cdot$ Urinary tract infection

\section{Impacts on practice}

- More than $50 \%$ of children presenting with urinary tract infection have uropathogens resistant to co-amoxiclav.

- Resistance to co-amoxiclav is associated with recurrent urinary tract infection and longer hospital stay in children and adolescents with urinary tract infection.

- Sensitivity to co-amoxiclav improves from $47.8 \%$ to $>96 \%$ when used in combination with gentamicin.

Montasser Nadeem

drnadeem.gad@gmail.com

1 Department of Paediatric, Children's Health Ireland at Tallaght University Hospital, Dublin, Ireland

2 Department of Microbiology, Tallaght University Hospital, Dublin, Ireland

3 Department of Radiology, Tallaght University Hospital, Dublin, Ireland

4 Trinity College Dublin, Dublin, Ireland

\section{Introduction}

Urinary Tract Infections (UTI) occur in up to 6-8\% of febrile infants and young children, with Escherichia coli (E. coli) being the commonest uropathogen [1]. Accurate diagnosis of UTI in children is important as there is a risk of renal scarring if left untreated. Conversely, the treatment of asymptomatic bacteriuria exposes patients to the potential adverse effects of antimicrobials and accelerates the development of resistance.

Urine culture is the gold standard for identifying the causative uropathogen and performing antimicrobial sensitivities to guide treatment. These results may take up to $48 \mathrm{~h}$ so the choice of empiric antibiotic therapy during this time is important. Numerous guidelines exist regarding this [2-6]. NICE guidelines recommend that for children $>3$ months old co-amoxiclav should only be used in combination with other drugs or if known to be susceptible [2]. American Academy of Paediatrics (AAP) guidelines recommend antimicrobial prescribing based on local antibiotic susceptibility 
data, however they make no recommendation regarding the empiric use of antibiotics in combination [3].

Unnecessarily antibiotic exposure could lead to emergence of antibiotic resistance. Indeed this occurs with commonly used empiric antibiotics, with a hypothesis that antibiotic resistance can occur in children using empiric antibiotics for recurrent UTI.

\section{Aim of the study}

The aim of this study was to examine the resistance rates of uropathogens to empiric co-amoxiclav as monotherapy and in combination with gentamicin. We also aimed to investigate whether there is a link between co-amoxiclav resistance and other variables such as recurrent UTI, patients' age at presentation, gender, and the presence of atypical UTI or radiological abnormalities in children and adolescents with UTI abnormalities.

\section{Study approval}

This was a retrospective review of the electronic patients records of patients admitted with UTI. Approval was obtained to perform this review.

\section{Methods}

A cohort of 209 patients with symptomatic UTI admitted to a paediatric hospital between January 2018 and December 2019 was identified from electronic patient records. Children with renal hypodysplasia, renal agenesis, multicystic dysplastic kidney, polycystic kidney disease, ureteropelvic junction obstruction or neural tube defects were excluded. A UTI was defined as the presence of typical symptoms in a child with a pure growth of an organism with $>10^{5}$ colony forming units per millilitre on urine culture. Antibiotic susceptibility data were obtained from the laboratory information system. Radiologic imaging results were also reviewed in order to identify any potential association between urinary tract abnormalities and antimicrobial resistance rates. Abnormal dimercaptosuccinic acid scintigraphy (DMSA) was defined as the presence of renal scarring or significant imbalance in renal uptake. Micturating cystourethrogram (MCUG) was defined as abnormal if vesicoureteral reflux (VUR) or renal tract obstruction was identified. Atypical and recurrent UTI were defined as per NICE guidelines (2).

Using SPSS version 27, summary measures were calculated and are reported as mean and standard deviation (SD). One-way ANOVA was used to explore the differences between means. Univariate and multivariate logistic regression models were used to estimate the association between the variables and the outcome. A $p$-value $<0.05$ was considered statistically significant.

\section{Results}

We examined 209 patients with UTI, the demographic data is presented in Table 1. The mean (SD) age was 23.73 (32.86) months, [range 5 days-13.93 years] and length of stay was 3.42 (2.13) days. Of 209 patients, 120 (57.4\%) were female, $190(90.1 \%)$ had E. coli identified as the causative pathogen and 176/209 (84.2\%), experienced their first UTI. In this cohort of patients, over half (109 of 209 (52.2\%)) had UTI due to uropathogens resistant to co-amoxiclav. In this cohort of patients, a review of sensitivities to alternative antibiotics demonstrated gentamicin sensitivity in 199/209 (95.2\%), cefuroxime sensitivity in 194/209 (92.8\%), cefalexin sensitivity in 189/209 (90.4\%), nitrofurantoin sensitivity in 207/209 (99.0\%) and trimethoprim sensitivity in $142 / 209$ (67.9\%). Notably, when co-amoxiclav in combination with gentamicin was analysed, overall susceptibility to both agents was $96.2 \%$ (201/209). Similarly, sensitivity to cefuroxime and cephalexin in combination with gentamicin was demonstrated in 207/209 (99\%) and 206/208 (99\%), respectively.

Microbial resistance to co-amoxiclav was associated with increased length of hospital stay with a mean (SD) of 3.9 (2.9) days compared to 3.1 (1.8) days in those who were susceptible ( $p$-value $<0.02)$. There was no association between co-amoxiclav resistance and patients' age ( $p$-value 0.52 ). Moreover, uropathogens were sensitive to co-amoxiclav in 43 of $89(48.3 \%)$ male patients and 57 of $120(47.5 \%)$ female individuals, with no association being reported between coamoxiclav resistance and patients' gender ( $\mathrm{p}$ value 0.91 ).

Table 1 Patient demographics including type of UTI and radiological findings

\begin{tabular}{lcc}
\hline & Number & Percent $(\%)$ \\
\hline Number of patients & 209 & \\
Female & 120 & 57.4 \\
$\begin{array}{l}\text { E. coli identified as the causative } \\
\text { pathogen }\end{array}$ & 190 & 90.1 \\
First UTI & 176 & 84.2 \\
Recurrent UTI & & 15.8 \\
Atypical UTI & 33 & 13.9 \\
UTI due to uropathogens resist- & 109 & 52.2 \\
$\quad$ ant to co-amoxiclav & 29 & \\
Renal ultrasound scan & & \\
$\quad$ Normal & 165 & 78.9 \\
Abnormal & 25 & 11.9 \\
Pyelonephritis & 19 & 9.1 \\
\hline
\end{tabular}

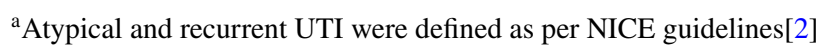


In this cohort of patients, a link between resistance to co-amoxiclav and recurrent UTI has been reported. While the majority [ 29 of $33(87.9 \%)$ ] of individuals with recurrent UTI had uropathogens resistant to co-amoxiclav, approximately less than half [80 of $176(45.5 \%)$ ] of patients with first UTI experienced the same resistance ( $p$ value 0.001 ). However whether UTI is typical or atypical has no impact on the antimicrobial resistance ( $p$ value 0.11 ). Uropathogens were resistant to co-amoxiclav in 98 of $180(54.4 \%)$ patients with uncomplicated UTI caused by E. coli. However this resistance was documented in 11 of 29 (37.93\%) patients with atypical UTI caused by $E$. coli and non-E. coli uropathogens in 10 and 19 patients, respectively.

In this cohort, no statistically significant difference in co-amoxiclav sensitivities was observed between children with normal radiological imaging and those with renal tract abnormalities detected on ultrasound scans. Of 209 patients who underwent renal ultrasound scan (RUSS), uropathogen resistance to co-amoxiclav was reported in 87/165 (52.3\%) patients with normal RUSS, 12/25 (48\%) with abnormal RUSS and 10/19 (52.6\%) with pyelonephritis ( $p$ value 0.37 ).

\section{Discussion}

Increasing antimicrobial resistance rates in children with UTI has been reported in the literature [7-11]. In previous studies in paediatrics with UTI, Stuliz et al. demonstrated an increasing trend in uropathogen resistance to commonly used empiric antibiotics such as amoxicillin or amoxicillin/ clavulanic acid [8]. Previous studies have demonstrated significant global variation in resistance rates $[8,9]$ though the overall prevalence of resistance is increasing [9]. In children admitted with urinary tract infection during a 12-year study period, high resistance rates have been noted for common empiric antibiotics, such as amoxicillin and cotrimoxazole [10]. Nevertheless, the association between unnecessarily antibiotic exposure and the emergence of antibiotic resistance has been reported [11]. It has been reported that up to $80 \%$ of antibiotics used in Europe are prescribed at primary care level [11].

In this cohort of patients, $52.2 \%$ had UTI due to uropathogens resistant to co-amoxiclav. However, when co-amoxiclav used in combination with gentamicin, the overall susceptibility to both agents increased to $96.2 \%$. Whether co-amoxiclav is an appropriate monotherapy antibiotic in children with UTI is explored in the available guideline [2]. In accordance with NICE guideline [2], in this group of patients with UTI, we observed that co-amoxiclav should be administered as monotherapy only in the context of known sensitivities, otherwise it should be given in combination where treatment is empiric. Moreover, our finding also supports that local antibiotics should be considered in prescribing antibiotics, as it was previously recommended [3].

The results from this cohort of patients support our hypothesis that there is a link between recurrent UTI and the microbial resistance to co-amoxiclav. Moreover, the association between increased length of stay and resistance to co-amoxiclav suggests that the initial empiric choice of antibiotic may have been inappropriate.

\section{Conclusion}

In conclusion, in this cohort of patients, approximately one in two children with UTI have uropathogens resistant to co-amoxiclav. However, one in 26 patients with UTI have microbial resistance to co-amoxiclav and gentamicin in combination. Notably, a link has been observed between resistance to co-amoxiclav and recurrent UTI, but not patients' age at presentation, gender, the presence of atypical UTI or radiological abnormalities. In this group of patients, the association between increased length of stay and resistance to co-amoxiclav suggests that the initial empiric choice of antibiotic may have been inappropriate. The results from this cohort of patients highlight that the current guidelines should be considered in the management of UTI and that antimicrobial prescribing should be based on local antibiotic susceptibility data.

Authors contributions JT, MN, AR P, MR: Contributed to study conception and design; JT, AR P, MH, MR: Contributed to data acquisition and interpretation; $\mathrm{MN}$ : contributed to data analysis and interpretation; All authors were involved in drafting and reviewing the manuscript; All authors have given final approval.

Conflicts of interest The auhtors declare that they have no conflict of interest. Funding (No specific funding was received)

Ethics approval Approval was obtained from our hospital ethics committee.

Open Access This article is licensed under a Creative Commons Attribution 4.0 International License, which permits use, sharing, adaptation, distribution and reproduction in any medium or format, as long as you give appropriate credit to the original author(s) and the source, provide a link to the Creative Commons licence, and indicate if changes were made. The images or other third party material in this article are included in the article's Creative Commons licence, unless indicated otherwise in a credit line to the material. If material is not included in the article's Creative Commons licence and your intended use is not permitted by statutory regulation or exceeds the permitted use, you will need to obtain permission directly from the copyright holder. To view a copy of this licence, visit http://creativecommons.org/licenses/by/4.0/. 


\section{References}

1. Kaufman J, Temple-Smith M, Sanci L. Urinary tract infections in children: an overview of diagnosis and management. BMJ Paediatr Open. 2019;3:e000487.

2. National Institute for Health and Care Excellence. Pyelonephritis (acute): Antimicrobial Prescribing. NICE guideline 111. 2018. https://www.nice.org.uk/guidance/ng111. Accessed Oct 122020.

3. Roberts KB. Urinary tract infection: clinical practice guideline for the diagnosis and management of the initial UTI in febrile infants and children 2 to 24 months. Pediatrics. 2011;128:595-610.

4. Stein R, Dogan HS, Hoebeke P, et al. Urinary tract infections in children: EAU/ESPU guidelines. Eur Urol. 2015;67:546-58 (European Association of Urology; European Society for Pediatric Urology).

5. 't Hoen LA, Bogaert G, Radmayr C, et al. Update of the EAU/ ESPU guidelines on urinary tract infections in children. J Pediatr Urol. 2021;17:200-7.

6. Ammenti A, Alberici I, Brugnara M, et al. Updated Italian recommendations for the diagnosis, treatment and follow-up of the first febrile urinary tract infection in young children. Acta Paediatr. 2020;109:236-47 (Italian Society of Pediatric Nephrology).
7. Edlin RS, Copp HL. Antibiotic resistance in pediatric urology. Ther Adv Urol. 2014;6:54-61.

8. Stultz JS, Doern CD, Godbout E. Antibiotic resistance in pediatric urinary tract infections. Curr Infect Dis Rep. 2016;18:40.

9. Erb A, Sturmer T, Marre R, et al. Prevalence of antibiotic resistance in Escherichia coli: overview of geographical, temporal and methodological variations. Eur J Clin Microbiol Infect Dis. 2007;26:83-90.

10. Bitsori M, Maraki S, Galanakis E. Long-term resistance trends of uropathogens and association with antimicrobial prophylaxis. Pediatr Nephrol. 2014;29:1053-8.

11. European Centre for Disease Prevention and Control. Key messages for primary care prescribers. 2009. https://antibiotic.ecdc. europa.eu/en/get-informedkey-messages/key-messages-primarycare-prescribers. Accessed 23 Nov 2020.

Publisher's Note Springer Nature remains neutral with regard to jurisdictional claims in published maps and institutional affiliations. 